\title{
Corrigendum: Selective inhibition of 2-AG hydrolysis enhances endocannabinoid signaling in hippocampus
}

Judit K Makara, Marco Mor, Darren Fegley, Szilárd I Szabó, Satish Kathuria, Giuseppe Astarita, Andrea Duranti, Andrea Tontini, Giorgio Tarzia, Silvia Rivara, Tamás F Freund \& Daniele Piomelli

Nature Neuroscience 9, 1139-1141 (2005); Published online 7 August 2005

Our paper identified 6-methyl-2-p-tolylaminobenzo[d] oxazin-4-one (URB754; Specs) as a monoacylglycerol lipase (MGL) inhibitor that enhances hippocampal depolarization-induced suppression of inhibition (DSI). However, in subsequent tests of non-commercial URB754, we failed to replicate these results, suggesting that a bioactive impurity was present in the commercial material. We have identified this impurity as bis(methylthio)mercurane (Supplementary Results online). Because this compound interacts with multiple targets, we tested another MGL inhibitor, methylarachidonylfluorophosphonate (MAFP), which prolonged DSI (Fig. 1), confirming that monoacylglycerol lipase contributes to the termination of DSI, as others have reported ${ }^{1}$. Another generation of endocannabinoid metabolism inhibitors is needed to test this hypothesis further.

Note: Supplementary information is available on the Nature Neuroscience website.

1. Szabo B et al. J. Physiol. (Lond.) 577, 263-280 (2006).
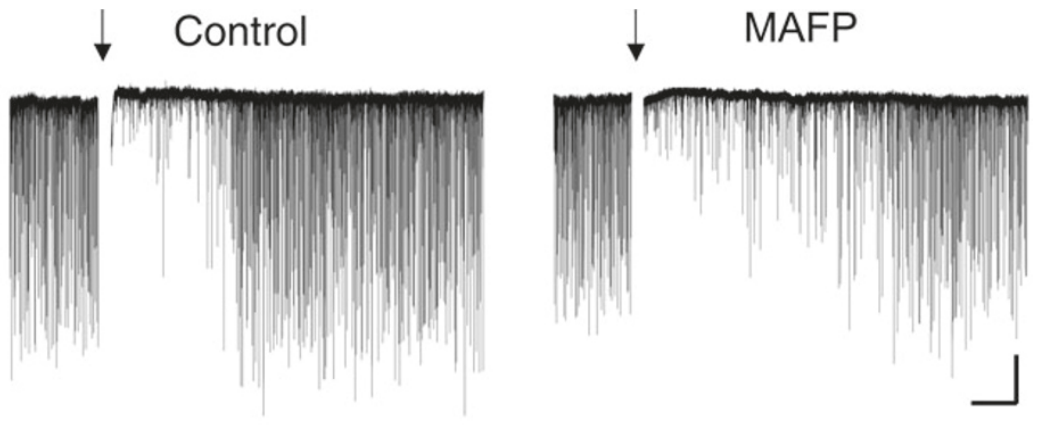

Figure 1 Effects of MAFP on DSI in hippocampal CA1 pyramidal cells. Top, traces from a representative experiment showing the effects of vehicle (ethanol, $0.00003 \%$ ) or MAFP (Tocris, $45 \mathrm{nM}$ ) on the transient reduction of spontaneous inhibitory postsynaptic potentials (IPSCs) elicited by a depolarizing stimulus (arrow). Scale bars, 100 pA, 5 s. Bottom left, averaged time-course of DSI after administration of vehicle (solid squares) or MAFP (open squares). Bottom right, DSI area in the first $30 \mathrm{~s}$ after stimulus application was significantly larger in MAFP-treated than in control slices.
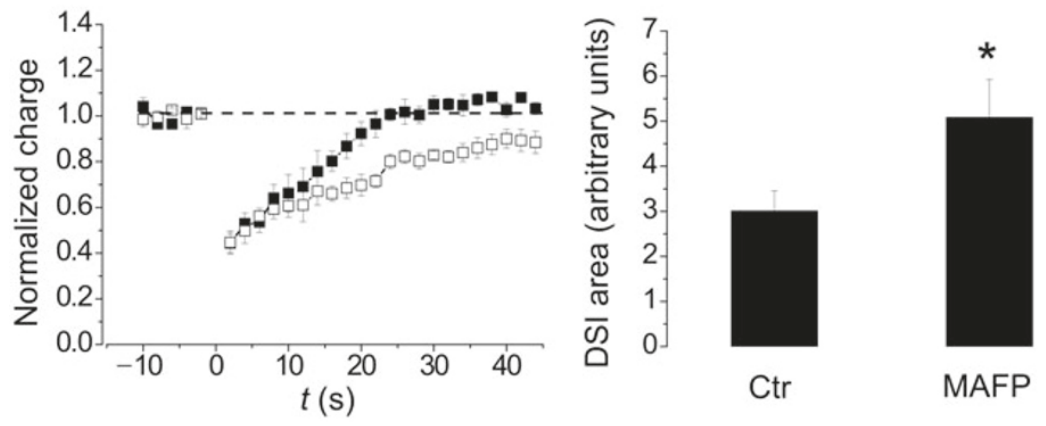

\section{Erratum: Reduced sodium current in GABAergic interneurons in a mouse model of severe myoclonic epilepsy in infancy}

Frank H Yu, Massimo Mantegazza, Ruth E Westenbroek, Carol A Robbins, Franck Kalume, Kimberly A Burton, William J Spain, G Stanley McKnight, Todd Scheuer \& William A Catterall

Nature Neuroscience 9, 1142-1149 (2006); published online 20 August; corrected after print 13 December 2006

In the version of this article initially published, the acceptance date was incorrect. The paper was accepted on 2 August 2006 . This error has been corrected in the PDF versions of the article.

\section{Erratum: The many roots of aggression}

Jordan Grafman, Maren Strezniak \& Frank Krueger

Nature Neuroscience 9, 1347 (2006); published online 26 October 2006; corrected after print 21 November 2006

In the version of this article initially published, the second author's name was spelled incorrectly. The correct name should be Maren Strenziok. The error has been corrected in the HTML and PDF versions of the article. 